\title{
Design of Novel Compound Fresnel Lens for High-Performance Photovoltaic Concentrator
}

\author{
Lei Jing, ${ }^{1,2}$ Hua Liu, ${ }^{1}$ Huifu Zhao, ${ }^{1,2}$ Zhenwu Lu, ${ }^{1}$ Hongsheng Wu, ${ }^{1}$ He Wang, ${ }^{1}$ and Jialin $\mathrm{Xu}^{1}$ \\ ${ }^{1}$ Opto-Electronics Technology Center, Changchun Institute of Optics, Fine Mechanics and Physics, Chinese Academy of Sciences, \\ Changchun 130033, China \\ ${ }^{2}$ Graduate University of the Chinese Academy of Sciences, Beijing 100039, China
}

Correspondence should be addressed to Hua Liu, liuhua_rain@yahoo.com.cn

Received 31 May 2011; Accepted 30 July 2011

Academic Editor: Songyuan Dai

Copyright () 2012 Lei Jing et al. This is an open access article distributed under the Creative Commons Attribution License, which permits unrestricted use, distribution, and reproduction in any medium, provided the original work is properly cited.

\begin{abstract}
We present a new design of compound Fresnel-R concentrator which is composed of two lenses: a primary lens (Fresnel lens) that works by total internal reflection at outer sawteeth but refraction at inner sawteeth, and a ringed secondary lens that works by refraction. In contrast to previous Fresnel lens concentrators, this design increases the acceptance angle, improves the irradiance uniformity on the solar cell, and reduces the aspect ratio significantly. Meanwhile several sawteeth of the primary Fresnel lens can correspond to a same ring of secondary lens, which will efficiently lower the complexity of designing and manufacturing. Moreover, in order to reduce the influence of manufacturing tolerances and to increase the optical efficiency further, the central part of the bottom of the secondary lens which directly adhered to the solar cell is designed as a cone-shaped prism to collect the sunlight that does not reach the solar cell. Finally, we provide simulations and analyses of the design method an optical efficiency more than $80 \%$ and an aspect ratio smaller than 0.5 can be achieved.
\end{abstract}

\section{Introduction}

The photovoltaic industry is growing rapidly today; however, it is still limited by the high cost of photovoltaic systems, especially the expensive semiconductor material. An effective way to reduce the cost is to cut down the amount of the semiconductor material by means of combination with concentrating optics. The Fresnel lens has been used as a concentrator in photovoltaic field for many years [1-3]. The main disadvantages of these concentrators are long focal distance and nonuniform illumination on the solar cell's active area. The first photovoltaic integrating concentrator was proposed by Sandia National Labs in the late 1980s [4], which used a Fresnel lens as the primary lens and an imaging single surface lens as the secondary lens to obtain the uniform irradiance distribution on the cell, and it was commercialized later by Alpha Solarco. This kind of concentrator consists of two imaging optical lenses, where the Fresnel lens is placed at the single surface lens' focal plane, and vice versa. Despite the simplicity and uniform illumination on cell of this concept, their application is still restricted to low concentrations $(<1000 \mathrm{x})$ due to its low acceptanceconcentration product.

In addition to the excellent performance of the multijunction solar cell under high concentration sunlight [5], a higher concentration factor can considerably reduce the cost of solar photovoltaic generation [6]. Thus, one of the purposes of our work is to design nonimaging Fresnel lenses used in concentrating photovoltaic systems (CPVs) with a high concentration factor but its aspect ratio (i.e., the ratio of focal length to the aperture of lens) maintains a relatively small value. For traditional Fresnel concentrator, however, the focal length of CPVs will be enlarged with the geometric concentration. A good strategy to increase the concentration of the Fresnel CPV system but not to enlarge the focus of the Fresnel lens is to design most of Fresnel lens's sawteeth as total internal reflectors (TIR) [7], whose aspect ratio can be even lower than 0.5 . Nevertheless, the closer to the centre of Fresnel lens, the more serious is its Fresnel loss resulting from the vertical facet, and the design method is relatively complex. Unfortunately, there are few investigations paying more attention to the determination of critical radius which 
can simplify the design method and the further increase of optical efficiency.

With this background, in this paper a more realistic design method is proposed to design a high concentration compound Fresnel lens with a small aspect ratio, which combined the outer sawteeth that work by total internal reflection with the inner sawteeth that work by refraction. The critical radius where the sawteeth of Fresnel lens begins to perform TIR is derived by using simple trigonometry. Furthermore, the approach of simplifying design method that several sawteeth of the primary Fresnel lens can correspond to a same ring of secondary lens is also presented. Thus, only several aspherical rings are designed as secondary lens' front surface is done in the CPVs to obtain more uniform irradiance distribution. Finally, a cone-shaped prism to collect the sunlight that does not reach the solar cell is also added to consummate the CPVs, which will increase the optical efficiency further.

\section{Design Method}

The CPV optics constitute a typical design problem that contains both the bundle coupling problem for obtaining maximum acceptance-concentration product and the prescribed irradiance to obtain uniform irradiance distribution on the solar cell area [4]. This is usually a difficult task and only the partial solutions have been found.

The design method includes two parts: design of primary Fresnel lens and design of secondary lens of CPV system. The former also includes two parts: design of the outer sawteeth of Fresnel lens that work by total internal reflection and design of the inner sawteeth that work by refraction. The front surface of secondary lens has several aspheric rings and is designed to optimize the spatial distribution of the light over the solar cell. The solar cell is adhered at the bottom of the secondary lens directly, making it simple to seal against moisture and prevent misalignment. Figure 1 shows the cross-sectional sketch of this compound Fresnel-R concentrator for CPV system. Incident light is assumed to be parallel and the circular solar cell is adopted as an example in the following design. Since the source and solar cell are both of axial symmetry, only one quarter must be considered in this discussion and the final device is obtained by applying the rotational symmetry.

2.1. Design of Primary Fresnel Lens. First, we design the outer part of primary Fresnel concentrator that works by total internal reflection. Before that we need to determine the critical radius which cuts the Fresnel lens into two different parts: the outer part that works by TIR and the inner part that works by direct refraction, as shown in Figure 1 (red dashed line). The closer to the centre of Fresnel lens, the more serious is the Fresnel loss resulted from the vertical facet, and the bigger is the size of sawtooth. Thus, it is not appropriate to design most of the sawteeth as TIR, especially for the centre sawteeth, which can be refractive sawteeth. Actually, there is a critical radius that determines which is suitable: TIR or direct refraction. For the direct refractive sawtooth (see, e.g., Figure 2(a)), we can deduce the relationship of incident angle

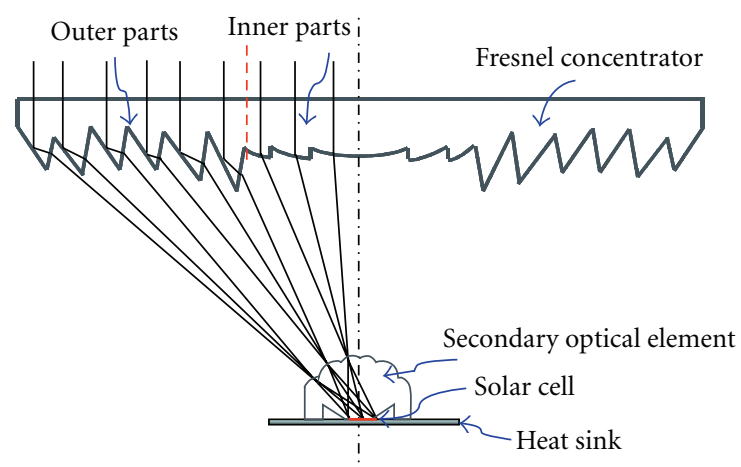

Figure 1: Sketch map of compound Fresnel-R lens for CPV system.

$i$ interrelated to Fresnel loss and convergent angle $\theta$ of the Fresnel lens:

$$
i=\arctan \left(\frac{\tan \theta}{n \sqrt{1+\tan ^{2} \theta}-1}\right),
$$

where $n$ is the refractive index of the Fresnel lens. And a similar formula for the TIR sawtooth can be calculated similarly (see, e.g., Figure 2(b)):

$$
i^{\prime}=\arcsin \left(\frac{1}{n \sqrt{1+\tan ^{2} \theta}}\right) \text {. }
$$

Figure 3 illustrates the convergent angle with the incident angle for TIR sawtooth (red curve) and direct refraction sawtooth (blue curve), which is obtained by (1) and (2). It noted that $x$-axis is $\tan \theta$, which is also approximately equal to $R_{n} / f$, where $R_{n}$ and $f$ are the radius of each sawtooth and the focal distance of Fresnel lens, respectively. The red line indicates that as the convergent angle $\theta$ reduces gradually (yellow arrow), the Fresnel loss caused by the refraction of the vertical facet will increase as opposed to the blue line. It is clear that there is a point of intersection between the red curve and the blue curve, which is the critical radius. Therefore, for the solutions left of the critical radius, it is more appropriate to design these sawteeth as direct refraction.

Achieving high reflectivity mirror is usually expensive and the degradation of the mirror quality could be faster than is desirable for a CPV system [8]. So, mirrors are not used in this design method. As shown in Figure 4(a), we expect that the rays (represented by red line) issuing from a planar wavefront $w$ could be total internal reflected by the sawtooth's titled surface, and then refracted by the sawtooth's vertical surface. Finally, the rays exiting from Fresnel lens could be focused on the point $F$. It is known that this is a conservation of the optical path length problem [9]. Considering a coordinate $(y, z)$ system, the optical path equation is expressed as

$$
\begin{gathered}
\mathrm{opl}=l_{1}+l_{2}+l_{3}, \\
l_{1}=n_{1} *|W P|=n_{1} * \sqrt{\left(W_{y}-P_{y}\right)^{2}+\left(W_{z}-P_{z}\right)^{2}},
\end{gathered}
$$




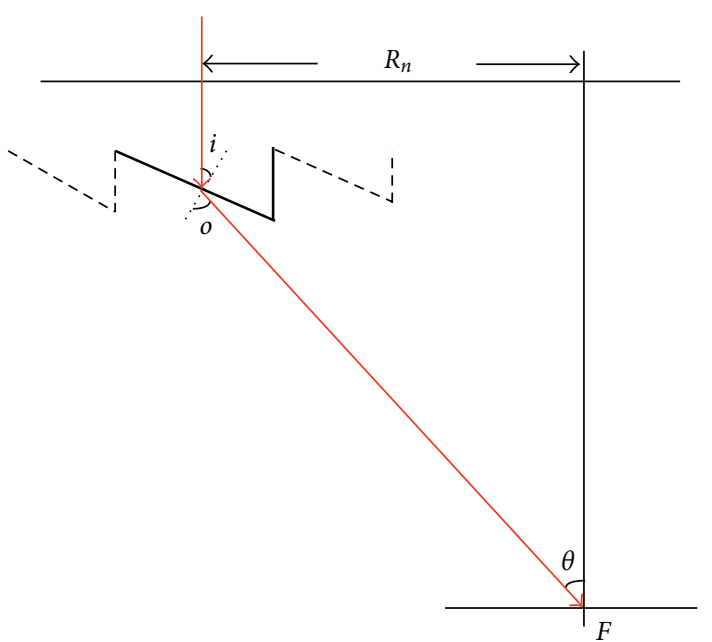

(a)

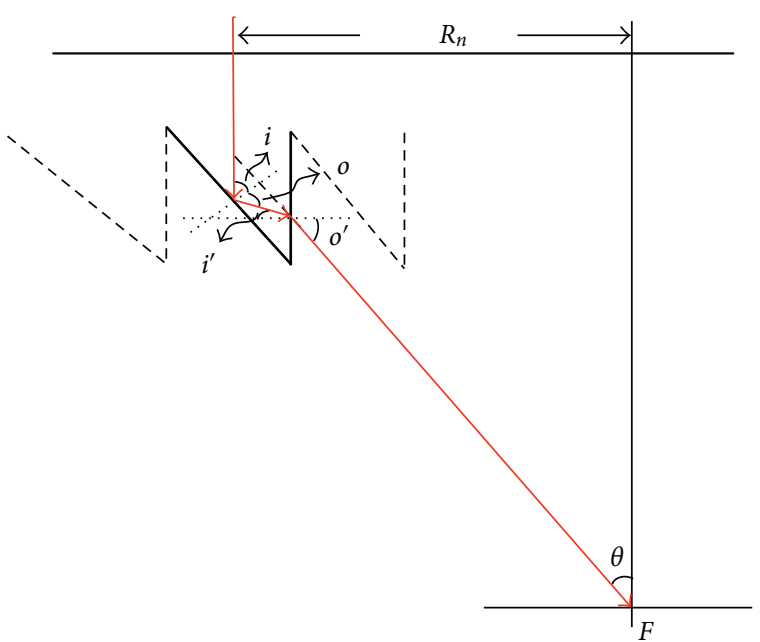

(b)

FIgure 2: Determination of the critical radius. (a) The direct refractive sawtooth. (b) The TIR sawtooth. The sawteeth and the angle of incident ray are locally exaggerated, only for interpreting conveniently.

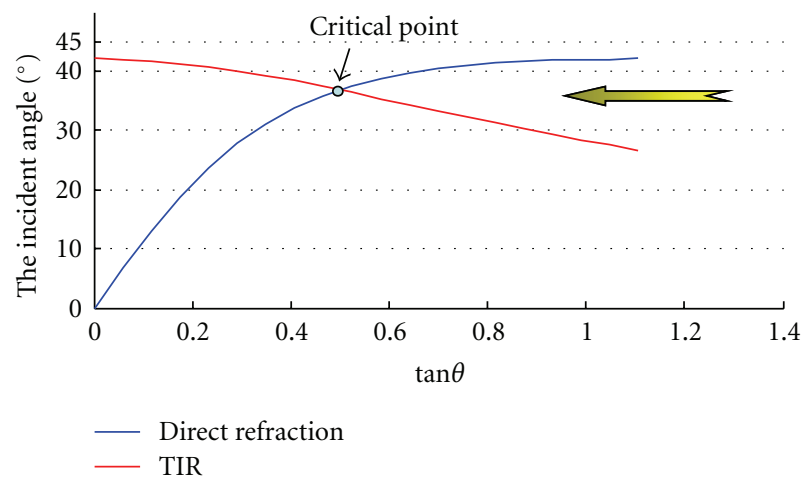

FIGURE 3: The change of incident angle $i$ with convergent angle $\theta$. The refractive index of Fresnel lens is adapted as $n=1.4918$.

$$
\begin{aligned}
& l_{2}=n_{1} *|P V|=n_{1} * \sqrt{\left(P_{y}-V_{y}\right)^{2}+\left(P_{z}-V_{z}\right)^{2}}, \\
& l_{3}=n_{0} *|V F|=n_{0} * \sqrt{\left(V_{y}-F_{y}\right)^{2}+\left(V_{z}-F_{z}\right)^{2}},
\end{aligned}
$$

where $n_{1}$ and $n_{0}$ are the refractive index of primary Fresnel lens and air, respectively, $l_{1}$ denotes the optical path length from the point $W$ (on the planar wavefront) to the point $P$ (on the tilted surface), $l_{2}$ denotes the optical path length from the point $P$ to the point $V$ (on the vertical surface), and $l_{3}$ denotes the optical path length from the point $V$ to the point $F$ (the focus of sawtooth). Once the lowest point $P_{1}$ of sawtooth, the planar wavefront $w$, and the focus of sawtooth $F$ are specified, the optical path length constant will be determined, and enough discrete points on the tilted surface of sawtooth can be acquired easily by the conservation of the optical path length expressed as (3) and the Snell's law expressed as

$$
\left[1+n^{2}-2 n(\vec{O} \cdot \vec{I})\right]^{1 / 2} \vec{N}=\vec{O}-n \vec{I}
$$

where $\vec{I}$ and $\vec{O}$ are the unit vectors of incident and refracted rays, $\vec{N}$ is the unit normal vector on the refracted point, and $n$ is the relative refraction index of Fresnel lens. The example examines only one sawtooth of the outer part of Fresnel concentrator; the others can be calculated in the same manner.

Secondly, we design the inner part of primary Fresnel concentrator that work by direct refraction. The design method is very similar to that of the outer sawtooth as described above. The only difference is that the rays (represented by red line) emitted from a plane wavefront $w^{\prime}$ are refracted but not totally internal reflected by the inner sawtooth as shown in Figure 4(b). Normally, we can also obtain enough discrete points on the curve of inner sawtooth uncomplicatedly by taking advantage of the conservation of optical path length equation and the Snell's law expressed as in the next equation and (4), respectively

$$
\begin{gathered}
\text { opl }=l_{1}+l_{2}, \\
l_{1}=n_{1} *\left|W^{\prime} P^{\prime}\right|=n_{1} * \sqrt{\left(W_{y}^{\prime}-P_{y}^{\prime}\right)^{2}+\left(W_{z}^{\prime}-P_{z}^{\prime}\right)^{2}}, \\
l_{2}=n_{0} *\left|P^{\prime} F^{\prime}\right|=n_{0} * \sqrt{\left(P_{y}^{\prime}-F_{y}^{\prime}\right)^{2}+\left(P_{z}^{\prime}-F_{z}^{\prime}\right)^{2}} .
\end{gathered}
$$

Note that in the calculation of these discrete points, the width of each sawtooth $t$ must be determined which is chosen before the design of primary lens, and then the lowest point $P_{n}$ of each sawtooth can be obtained. Until now, quite a number of discrete points on each curve of sawtooth have been obtained, therefore the final curve of each sawtooth is accomplished by appropriately fitting or interpolating these discrete points with a lower polynomial within the permissible error range.

2.2. Design of Secondary Lens. The design method of secondary lens is depicted in cross-section in Figure 5. For 


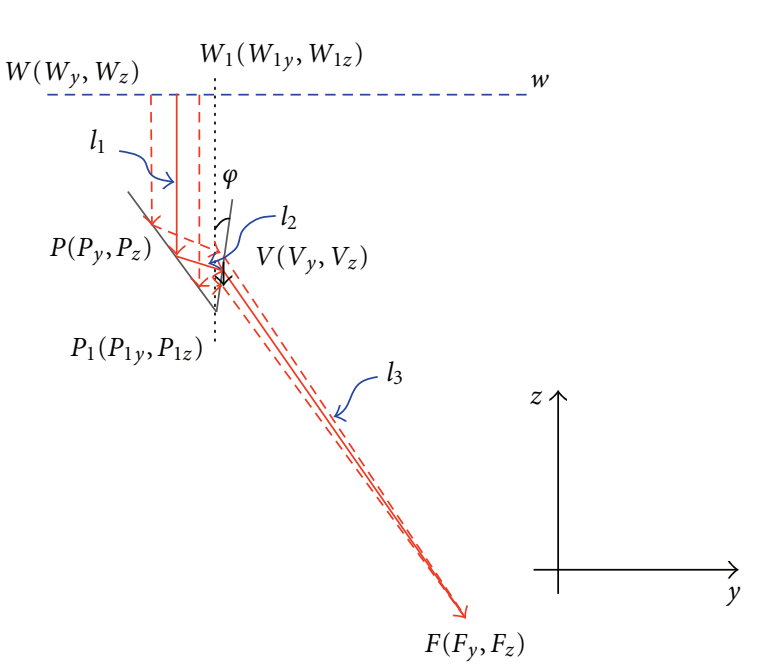

(a)

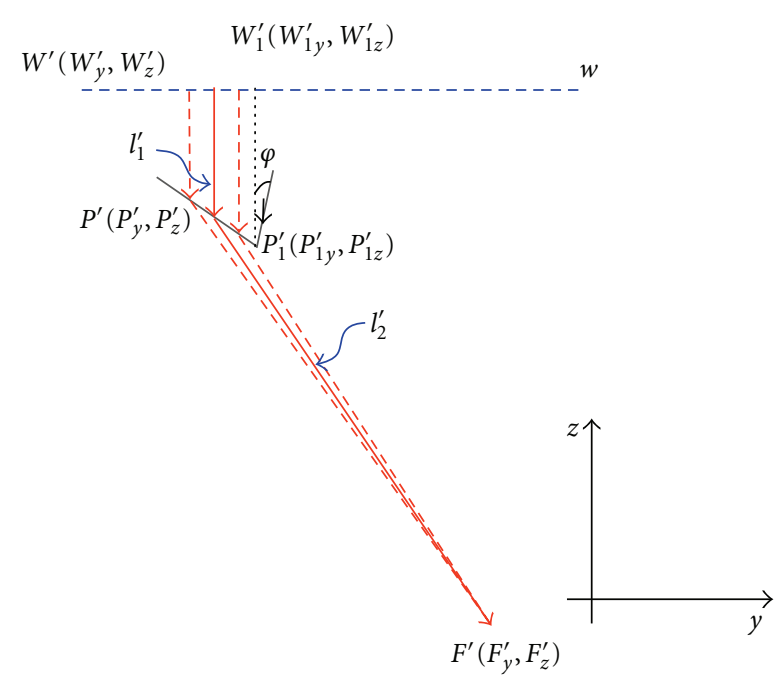

(b)

Figure 4: Design of the outer and inner sawteeth of primary Fresnel lens. The coordinate system is specified $(y, z)$, where $\varphi$ is the angle between the vertical surface and $z$-axis also named drag angle.

the convenience of interpretation, only one ring of secondary lens corresponding to one sawtooth of primary lens is shown. Actually, there is not a one-to-one correlation between a sawtooth of primary Fresnel lens and a ring of secondary lens, in other words, several sawteeth of primary Fresnel lens can correspond to one ring of secondary lens, as shown in Figure 1, three sawteeth directly correspond to one ring of the secondary lens, which can make the CPVs more convenient to design and manufacture the secondary lens. According to edge ray principle [6], rays from the edge of source should strike the edge of the target. Thus each portion of the front surface of secondary lens can be a Cartesian oval [10]. For example, one ring of secondary lens which is a Cartesian oval transform the edge ray bundle coming from point $P_{1}$ into the edge ray bundle impinging on the $R_{2}$ of the solar cell, as shown in Figure 5(a). With this it is forced that the other incident angle ray bundle can also reach the cell. The optical path of these rays is determined by the points $P_{1}, F, R_{2}$, and the refractive index of the dielectric material chosen at the beginning. Thus it is easy to determine this Cartesian oval with two focus $P_{1}, R_{2}$, and its fixed point $F$.

Next we need to calculate the borders of this ring of secondary lens' surface tracing the edge rays $r_{1}, r_{2}$ coming from positive and negative inner acceptance-angle $\theta_{i}$ which can be determined by the simple format of Snell's law expressed as equation:

$$
\sin \theta_{a}=n_{1} \sin \theta_{i},
$$

where $\theta_{a}$ and $\theta_{i}$ are the acceptance-angle of CPVs and the inner acceptance-angle, respectively. In the Figure 5(b), the intersections $S_{1}, S_{2}$ of positive acceptance-angle ray $r_{1}$ and negative acceptance-angle ray $r_{2}$ with the Cartesian oval are just the borders of the ring of secondary lens' surface.

Finally, in order to reduce the influence of manufacturing precision, a cone-shaped prism to collect the sunlight that does not reach the solar cell is added to consummate the CPVs, as shown with bold lines in Figure 6, the optical efficiency of total CPVs will be further improved. The angle $\alpha$ of incline $T$ can lie with respect to the horizontal is in the range of $20^{\circ} \sim 30^{\circ}$; however, any other suitable angle will work as long as most of solar ray $r$ can be reflected to the solar cell at the incline $T$.

\section{Example and Discussions}

Considering the cost of the manufacture of a real lens, numerical simulation based on the most widely used Monte Carlo ray tracing optical software is an efficient way to validate the concentrator design. A simple high concentration circular Fresnel lens was designed and simulated to validate this novel design method. The specifications of the design parameters are diameter of Fresnel lens $51 \mathrm{~mm}$, focus of Fresnel lens $21 \mathrm{~mm}$, drag angle is $2^{\circ}$, diameter of secondary lens $8.6 \mathrm{~mm}$, and diameter of solar cell $2 \mathrm{~mm}$. The geometrical concentration is $625 \mathrm{x}$ and the aspect ratio of the entire optical system is smaller than 0.5 . For this study we choose PMMA as the base Fresnel lens material for the optics. This is a very good option: high transmission factor, susceptible of molding manufacturing and with low cost. However, it is not suitable to be as the material of the secondary lens due to high temperature resulted from the high concentration, which will distort the plastic material. Thus the glass material of BK7 is adopted as the secondary lens material. The width of each groove is about $3 \mathrm{~mm}$, which can be freely adjusted due to manufacturing condition.

As shown in Figure 1, in our example, the primary Fresnel lens has six TIR sawteeth and two refractive sawteeth. The secondary lens has three aspherical rings. The first three TIR sawteeth of primary Fresnel lens correspond to the first ring of secondary lens and the secondary three TIR sawteeth correspond to the secondary ring. The two direct refraction 


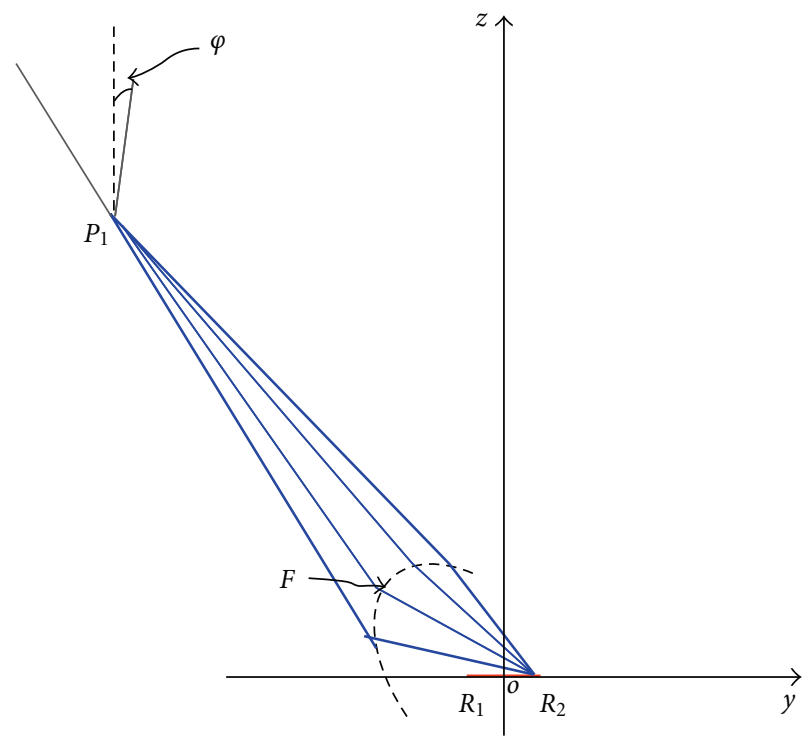

(a)

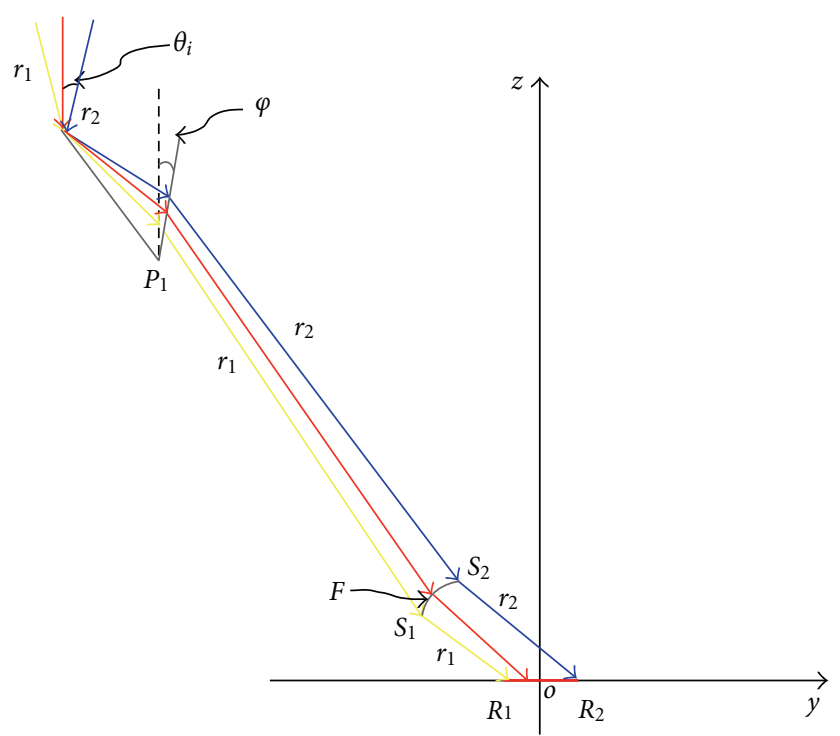

(b)

FIGURE 5: Design of secondary lens. (a) Specification of the type of secondary lens' front surface. (b) Calculation of the borders of secondary lens' front surface.

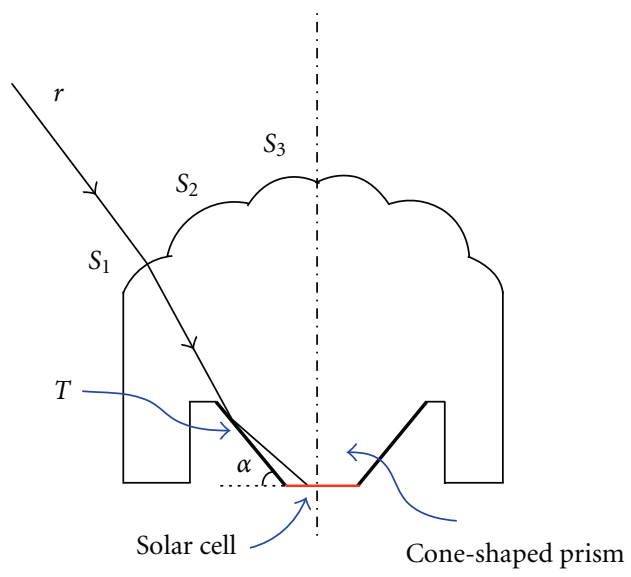

FIGURE 6: Schematic drawing of the secondary lens whose $T$ surface is designed as cone shaped.

sawteeth and a centre lens correspond to the tertiary ring. The profile of compound Fresnel lens for CPVs is calculated by the method mentioned above (see, e.g., Figure 7(a)), and its 3-dimension model is also created in CAD software: SolidWorks. And then we imported the 3-dimension solid into the widely used currently commercialized optical software, such as TracePro in this paper, for ray tracings of the optical system (see, e.g., Figure 7(b)).

This simulation was done by assuming that the refractive index of material PMMA and BK7 are 1.49 and $1.52(\lambda=$ $546.1 \mathrm{~nm}$ ), respectively, and the solar flux exists as parallel rays with a divergence angle of $0.27^{\circ}[7,8]$. The computer simulations of ray tracings use 2 million rays. The simulations of two traditional Fresnel concentrators without secondary lens and with secondary lens was also done to

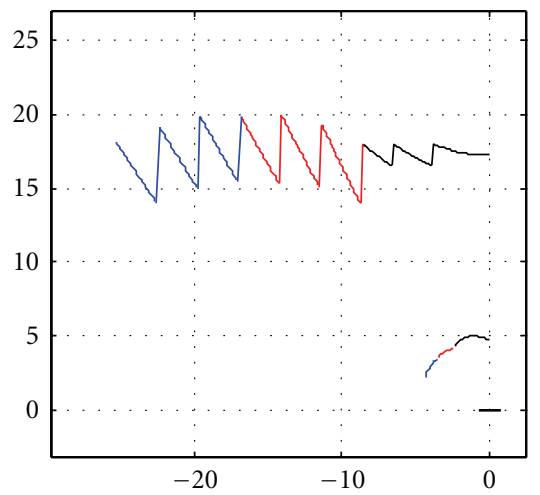

(a)

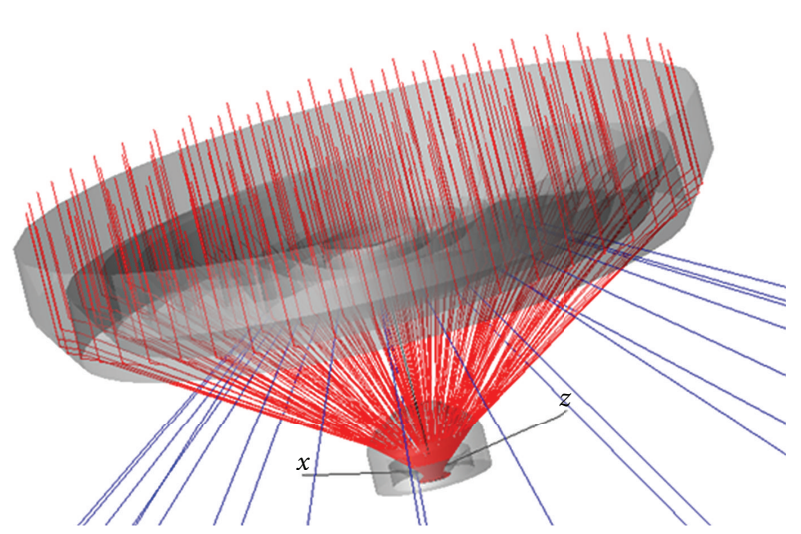

(b)

Figure 7: Profile and 3D cutaway view of a compound Fresnel-R lens for CPV system. 


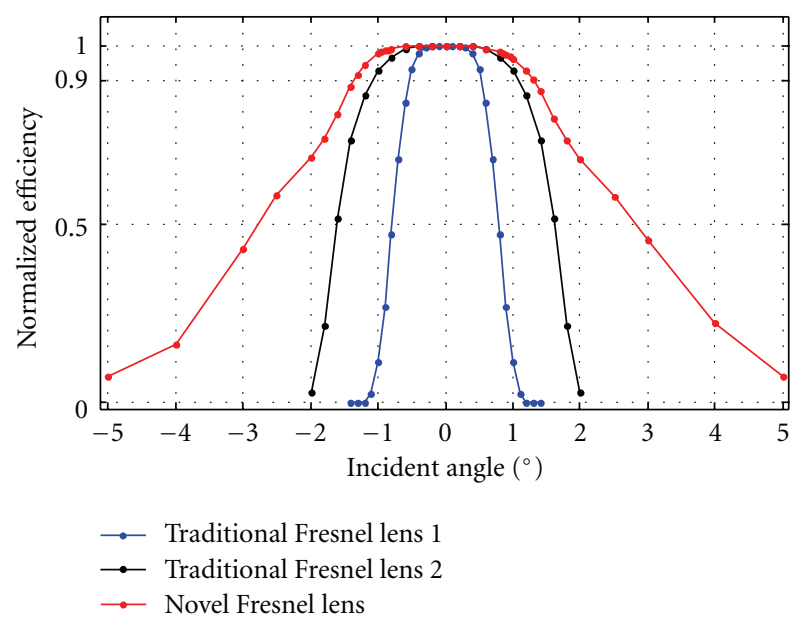

Figure 8: Relative transmission curve versus incident angle. The traditional Fresnel lens 1 and 2 represent the Fresnel concentrator without secondary lens and with secondary lens, respectively.

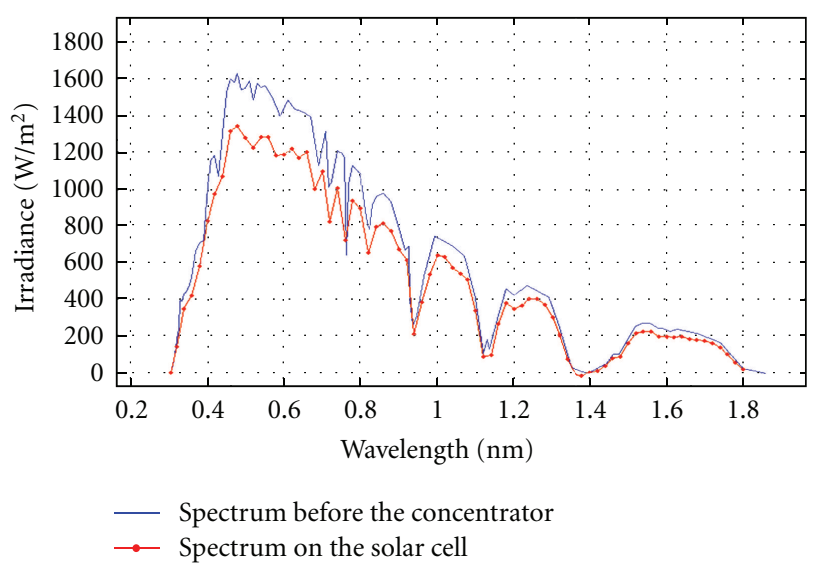

FIGURE 9: Spectral transmission power before and after the concentrator.

compare. Both the traditional Fresnel concentrators have an equivalent concentration. Based upon these simulations the novel Fresnel concentrator is found to have a 90\% acceptance angle of \pm 1.3 degrees (see, e.g., Figure 8 ). It is much better than the traditional Fresnel concentrator without a secondary lens, whose acceptance angle is always smaller than \pm 1 degrees. Although the acceptance angle of the traditional Fresnel concentrator with a secondary lens is close to the novel concentrator, its aspect ratio (1.2) is almost twice bigger than the novel concentrator (0.5).

As depicted in Table 1, the final optical efficiency received on the solar cell at normal incidence is even more than $82 \%$. Clearly, the main losses of this new optical system come from the Fresnel loss (e.g., reflection at the material/air interface), the sum of which is up to about $12.73 \%$. The secondary is the shadow loss that occur at the vertical facets of primary Fresnel lens, which is about $4.5 \%$. The Figure 9 shows the spectral transmission power of the designed optics system from $300 \mathrm{~nm}$ to $1800 \mathrm{~nm}$ : near $82.6 \%$ efficiency is achieved in the spectral range for triple-junction solar cells under solar

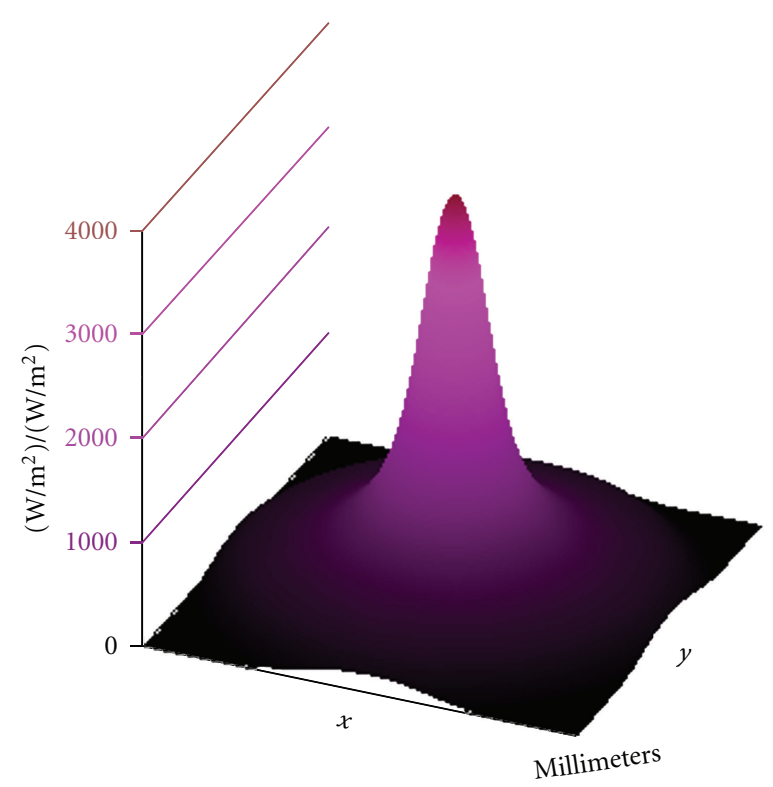

(a)

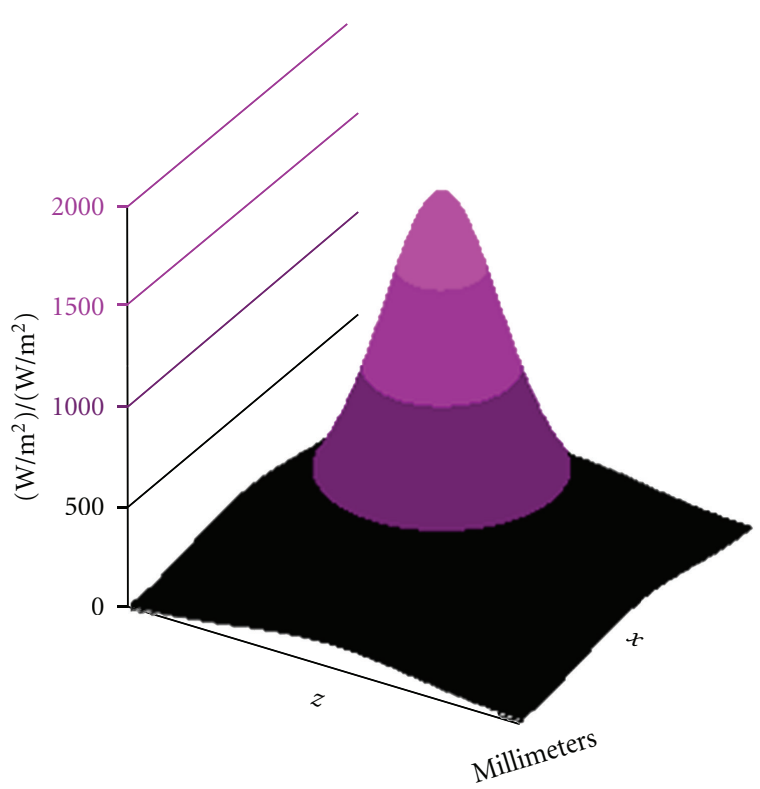

(b)

Figure 10: 3D irradiance distribution on the cell when the sun ray is at normal incidence. (a) Traditional Fresnel lens. (b) Novel compound Fresnel lens.

direction normal radiation (AM1.5D) due to the inclination angle, $T$, of the secondary lens.

In $3 \mathrm{D}$ the irradiance distribution is relatively uniform in the radial direction but not in the azimuthal direction because of its rotational symmetry. The simulation results are shown in Figure 10 when the sun ray at normal incidence. Note that the "irradiance" in Figure 10 is not the real irradiance value, it is the ratio of the real irradiance to the standard solar direction radiation $\left(1000 \mathrm{~W} / \mathrm{m}^{2}\right)$. The maximum local irradiance value is below 2000x (see, e.g., 
TABLE 1: The main losses of this concentrator system, considering the normal incidence and divergence angle of solar.

\begin{tabular}{|c|c|c|}
\hline The reason of losses & Losses & Transmission \\
\hline $\begin{array}{l}\text { Fresnel losses on the front surface of } \\
\text { primary Fresnel lens }\end{array}$ & $-3.94 \%$ & $96.06 \%$ \\
\hline $\begin{array}{l}\text { Fresnel losses on the sawteeth surface of } \\
\text { primary Fresnel lens }\end{array}$ & $-5.24 \%$ & $90.82 \%$ \\
\hline $\begin{array}{l}\text { Losses due to the drag angle of primary } \\
\text { Fresnel lens' sawteeth }\end{array}$ & $-4.50 \%$ & $86.32 \%$ \\
\hline $\begin{array}{l}\text { Fresnel losses on the surface of secondary } \\
\text { lens }\end{array}$ & $-3.55 \%$ & $82.77 \%$ \\
\hline
\end{tabular}

Figure 10(b)), which is better than the traditional Fresnel lens with a secondary design: 4000x (see, e.g., Figure 10(a)), and the uniformity is still sufficient to prevent the local peak irradiance from enlarging to deteriorate the solar cell.

\section{Conclusions}

A novel design of the compound Fresnel concentrator for CPV system was presented to increase the concentration factor, reduce the aspect ratio, and improve the optical performance on the solar cell. The simulation results have identified that using this method to design Fresnel concentrator, the aspect ratio (/ $f$ number) can be less than 0.5 , the optical efficiency of the optical system can be obtained more than $80 \%$ and the uniformity of irradiance on the solar cell can also be controlled to guarantee the good performance for the solar cell. All these factors will be helpful to substantially enhance the performance of CPVs and reduce its related cost when it is mass installed. The concentration module based on this novel compound Fresnel lens is a promising option for the development of a cost-effective photovoltaic solar energy generation.

\section{Acknowledgments}

The authors gratefully acknowledge the financial support from National Basic Research Program of China with Grant no. 2010CB227101 and the Innovation Program of Chinese Academy of Sciences.

\section{References}

[1] A. L. Luque and V. M. Andreev, Concentrator Photovoltaics, Springer, New York, NY, USA, 2007.

[2] R. Leutz and A. Suzuki, Nonimaging Fresnel Lenses: Design and Performance of Solar Concentrators, Springer Series in Optical Sciences, Springer, New York, NY, USA, 2001.

[3] E. Lorenzo and A. Luque, "Fresnel lens analysis for solar energy applications," Applied Optics, vol. 20, no. 17, pp. 29412945, 1981.

[4] L. W. James, "Use of refractive secondaries in photovoltaic concentrators," Tech. Rep. SAND89-7029, Sandia National Laboratories, Alburquerque, NM, USA, 1989.
[5] N. H. Karam, R. R. King, B. T. Cavicchi et al., "Development and characterization of high-efficiency Ga0.5In0.5P/GaAs/Ge dual- and triple-junction solar cells," IEEE Transactions on Electron Devices, vol. 46, no. 10, pp. 2116-2125, 1999.

[6] R. Winston, J. C. Miñano, and P. Benítez, Nonimaging Optics, Elsevier-Academic Press, New York, NY, USA, 2005.

[7] M. Hernández, A. Cvetkovic, P. Benítez, and J. C. Miñano, "High-performance Köhler concentrators with uniform irradiance on solar cell," in 5th Nonimaging Optics and Efficient Illumination Systems, Proceedings of the SPIE, August 2008.

[8] J. L. Alvarez, V. Díaz, and J. Alonso, "Optics design key points for high gain photovoltaic solar energy concentrators," in 2nd Optical Design and Engineering, Proceedings of the SPIE, September 2005.

[9] J. M. Born and E. Wolf, Principle of Optics, Pegamon, Oxford, UK, 1975.

[10] O. N. Stavroudis, The Mathematics of Geometrical and Physical Optics, John Wiley \& Sons, New York, NY, USA, 2006. 


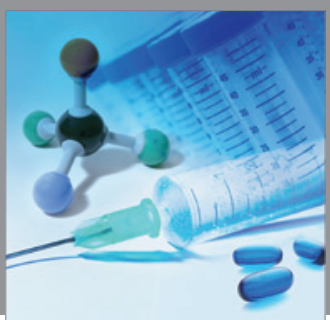

International Journal of

Medicinal Chemistry

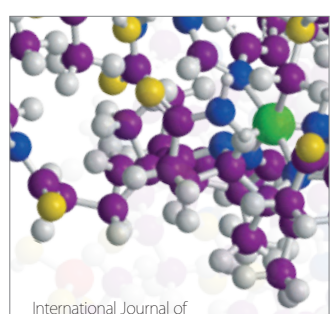

Carbohydrate Chemistry

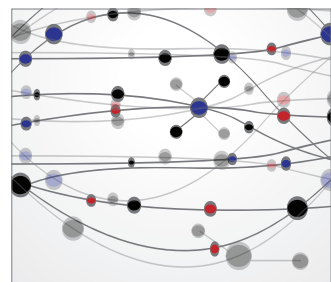

The Scientific World Journal
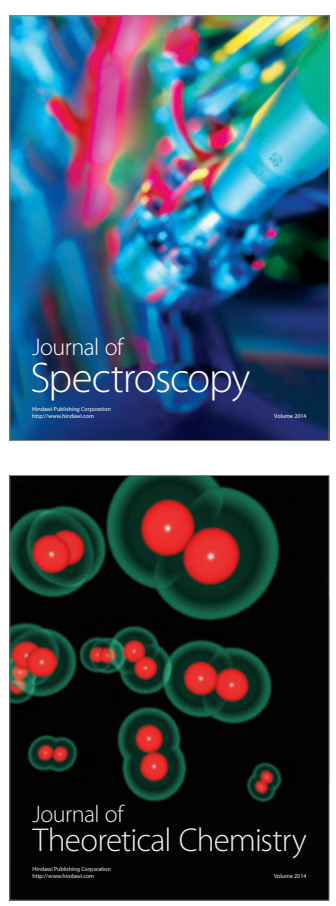
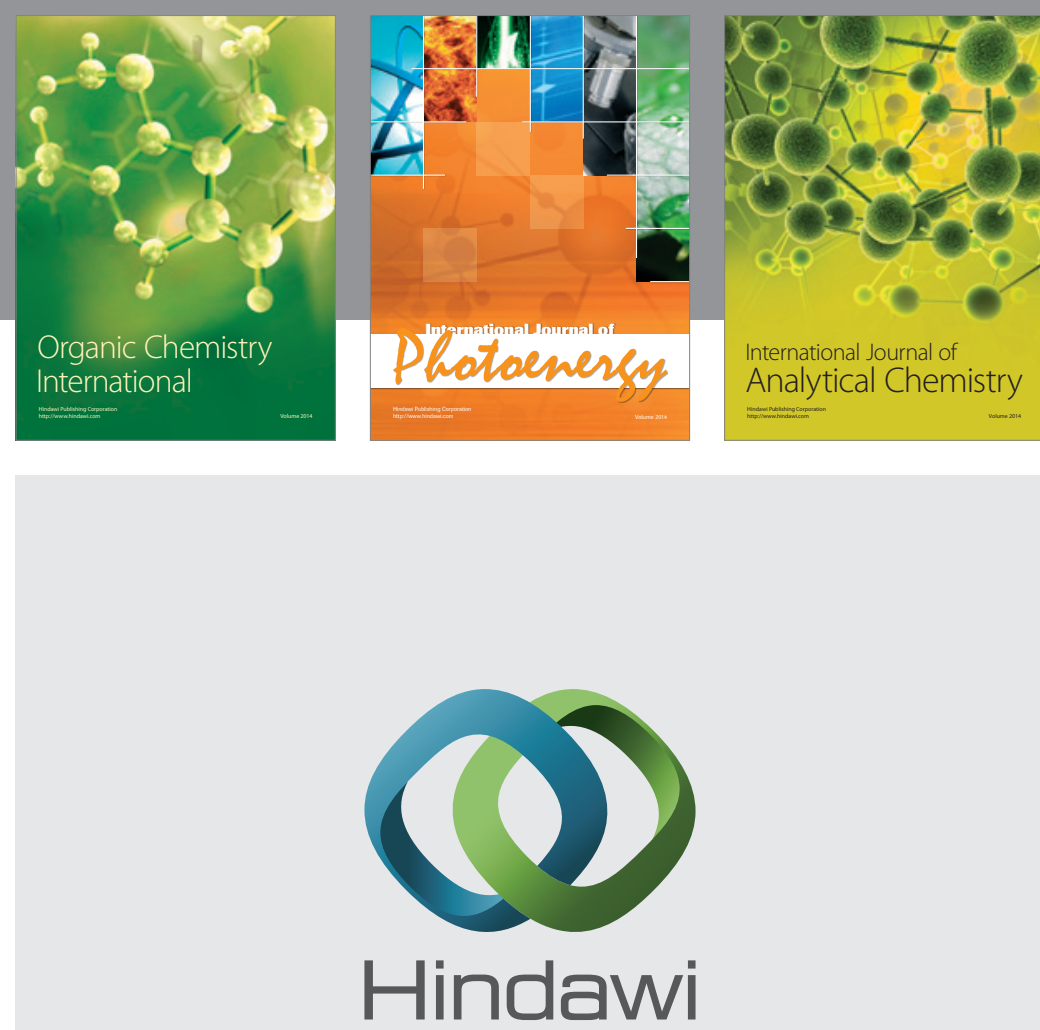

Submit your manuscripts at

http://www.hindawi.com
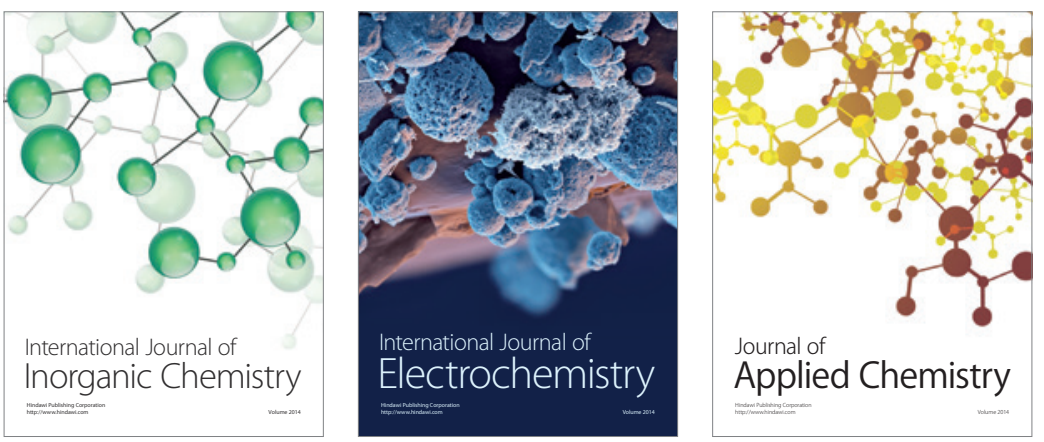

Journal of

Applied Chemistry
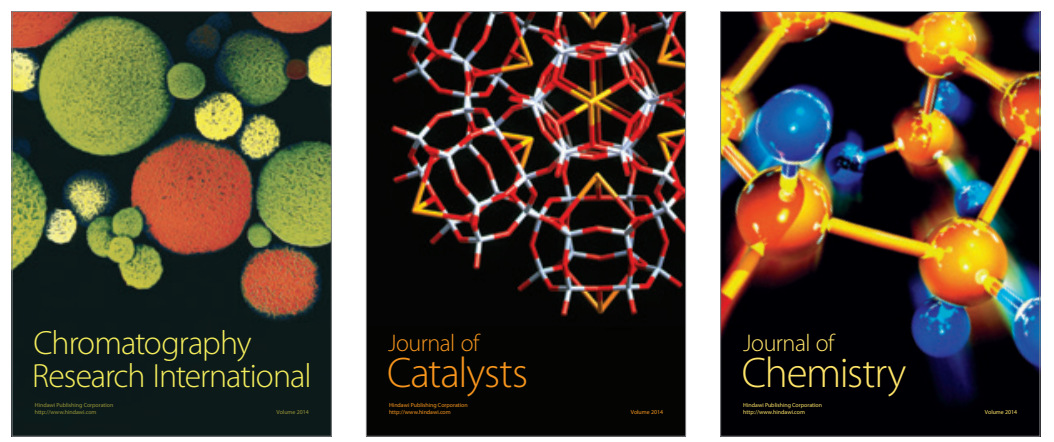
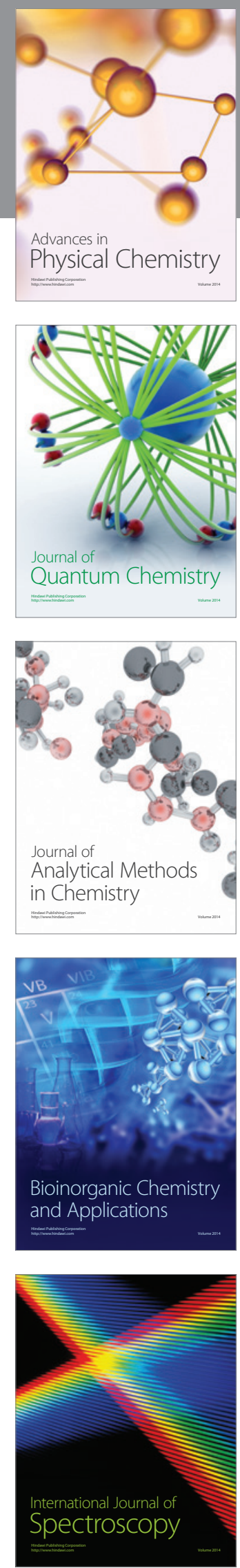